\title{
Visual Nonlinear Feedback Control of Liquid Level in Mold Sprue Cup by Cascade System with Flow Rate Control for Tilting-Ladle-Type Automatic Pouring System
}

\author{
Atsushi Ito ${ }^{1, a}$, Philipp Oetinger ${ }^{2}$, Ryosuke Tasaki ${ }^{1}$, Oliver Sawodny ${ }^{2}$, \\ and Kazuhiko Terashima ${ }^{1}$ \\ ${ }^{1}$ Toyohashi University of Technology, 1-1 Hibarigaoka, Tempaku, Toyohashi, Aichi, 441-8580, Japan \\ ${ }^{2}$ Institute of System Dynamics (ISYS), University Stuttgart, Waldburgstr. 17/19, 70563 Stuttgart, \\ Germany \\ aa_ito@syscon.me.tut.ac.jp
}

Keywords: casting industry, tilting-ladle-type automatic pouring system, nonlinear control, cascade control

\begin{abstract}
This paper presents the novel approach of liquid level control of mold sprue cup using camera information for tilting-ladle-type automatic pouring system in casting industry. A liquid level in mold sprue cup should be kept at high position to avoid inclusion of oxides and dusts during pouring process. A cascade control system with double feedback loop to enhance control response was proposed for the present level control: inner loop was the flowrate control system for pouring ladle, and outer loop was the level control one with camera measurement to detect the liquid level. The control part of flow rate was constructed based on the past study. The mathematical model of liquid behavior in a sprue cup of an inverted cone shape was built in this paper. The level controller was newly designed based on nonlinear control theory by exact linearization. The effectiveness of the proposed cascade system was verified by control simulations.
\end{abstract}

\section{Introduction}

As well known, casting is the metal working process involved phase transformation. In this process, molten metal is poured into mold and formed desired into the shape by solidification. This process can form complex shape by integral modeling and work various alloys which are difficult to produce in other processes. On the other hands, casting factoreis are severe place for workers, i.e. one of 3D jobs (dirty, difficult, and dangerous) since molten metal has high temperature more than $1000{ }^{\circ} \mathrm{C}$ and powder dust is caused by demolishing molds. Also, the pouring process is needed high level of skills to keep producing high quality casting products because a filling condition of molten metal in a mold depends on pouring flow rate. Thus, in order to reduce risks and improve the accuracy of products, advances have been made in the automation of the pouring process[1][2].

There have been some approaches to automate pouring process, such as tilting-type[3]-[8], stoppertype[9][10], and pressure-type[11]. In East Asia, the tilting-type automatic pouring approach is the main stream. The tilting-type approach has several advantages over other approaches, including the possibility of applying the skill of hand pouring and a mechanism that is easier to maintain. In the pouring process, it is important to control the outflow of molten metal from a ladle and pour into the correct position of the sprue cup in a mold. And, liquid level in sprue cup also should be kept at heigher point not to flow oxides or dusts into the product cavity of mold. The feedfoward approach has been proposed to control liquid level in former studies of our group[3]. In this approach, however, it is impossible to control liquid level properly if there are modeling error of mold shape and molten metal.

Therefore, the present study aims to control flow rate from ladle and liquid level in mold sprue cup using feedback control by visual measurement at mold sprue cup. In this paper, the cascade control 
system was proposed to achive exact liquid level control by using flow rate control at inner-loop to enhance the response of liquid level control. This system was verified by control simulations.

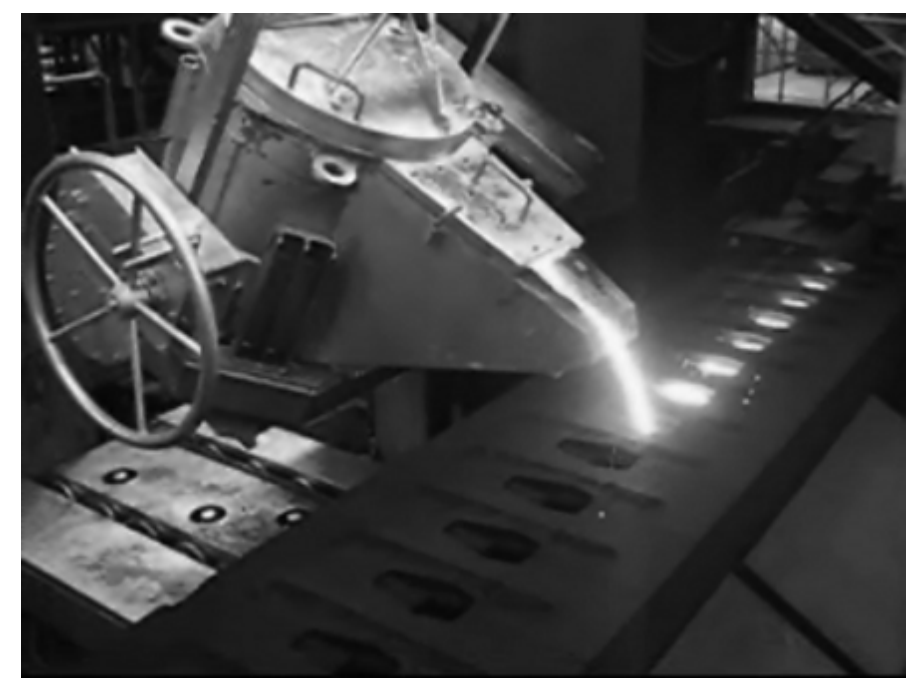

Fig. 1: Tilting-Type Automatic Pouring System

\section{Tilting-Type Automatic Pouring System}

An illustration of the automatic pouring machine used in the present work is shown in Fig. 2. A ladle is controlled by servo motors: Y-axis for backward and forward direction and Z-axis for down and up direction. And a ladle is tilted on $\Theta$-axis. The movable range of each motor is limited; Y-axis $0.4 \mathrm{~m}$, Z-axis $0.6 \mathrm{~m}$, and $\Theta$-axis: $\pm 90 \mathrm{deg}$ ( $0 \mathrm{deg}$ is defined as a horizontal posture of a ladle). A load cell built into a support member of the ladle, measures the total weight. Each system of motors receives velocity references from a Digital Signal Processor (DSP) and drives based on references. The sampling time of the DSP was $0.01 \mathrm{~s}$, which is a sufficiently small period for the pouring process control. Since the position of the pouring mouth generally moves by a conventional tilting motion, falling trajectory of outflow liquid varies. To stabilize the falling trajectory, the position of the pouring mouth was fixed by the synchronized control of $\mathrm{Y}$ and $\mathrm{Z}$-axes with the tilting motion of $\Theta$-axis, and incallled virtual nozzle[4]. In this study, the camera measurement equipment was embeded with image processing systems to verify and measure liquid level in mold sprue cup.

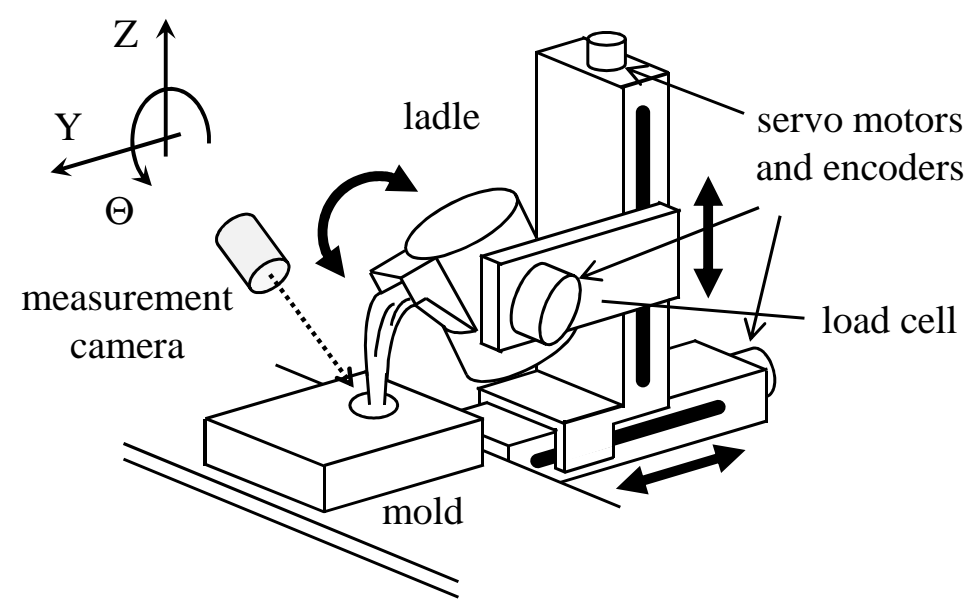

Fig. 2: System Configuration 


\section{Mathematical Models of Pouring Process}

The diagram of pouring process models is shown in Fig. 3[5]. The flow rate of tilting ladle is related with its angular velocity, and the tilting motion of ladle is controlled by $\Theta$-axis motor. The following paragraphs show details of process models of pouring process.

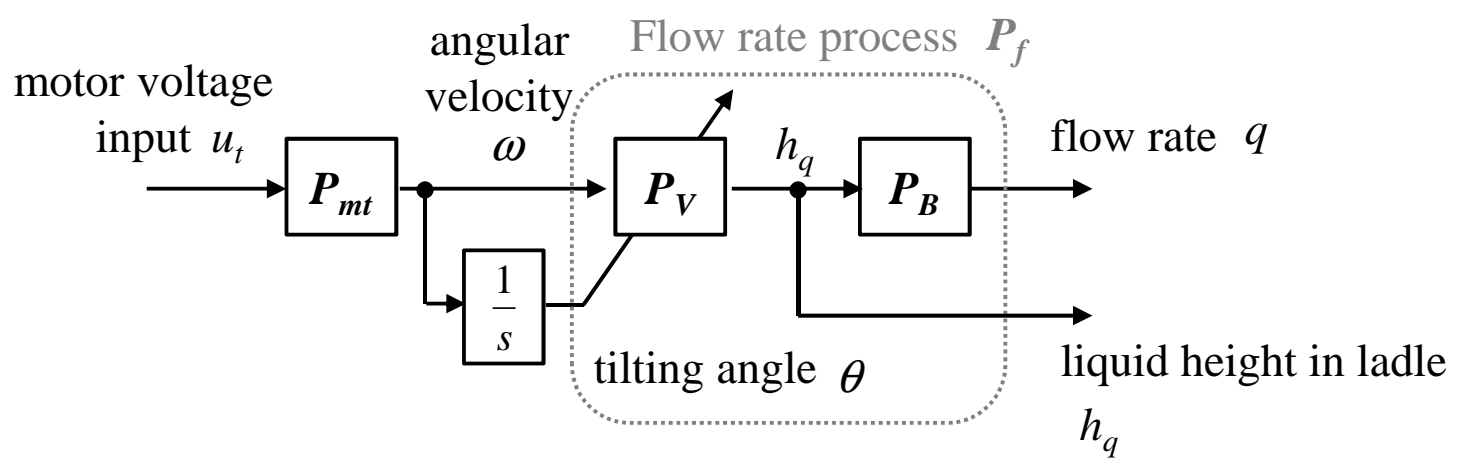

Fig. 3: Diagram of Pouring Process Models

Motor Model on $\Theta$-axis: $P_{t}$. The relationship between the input voltage $u_{t}$ and the tilting velocity $\omega$ is represented by the following first-order lag system,

$$
\dot{\omega}=-\frac{1}{T_{t}} \omega+\frac{K_{t}}{T_{t}} u_{t}
$$

where $T_{t}$ is the time constant, and $K_{t}$ is the gain. In this paper, $T_{t}=6.00 \times 10^{-3} \mathrm{~s}$, and $K_{t}=24.58 \mathrm{deg} /(\mathrm{s} \cdot \mathrm{V})$. The tilting angle of a ladle $\theta$ is derived from a time integration of $\omega$.

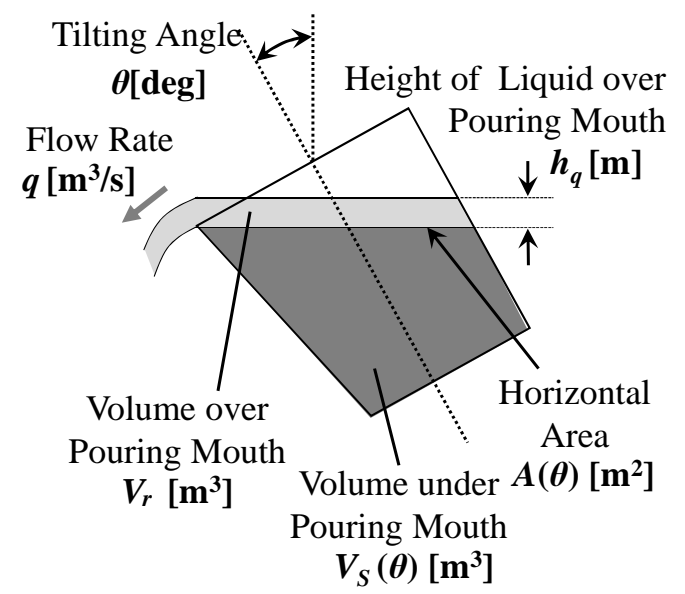

Fig. 4: Geometric parameters of volume balance

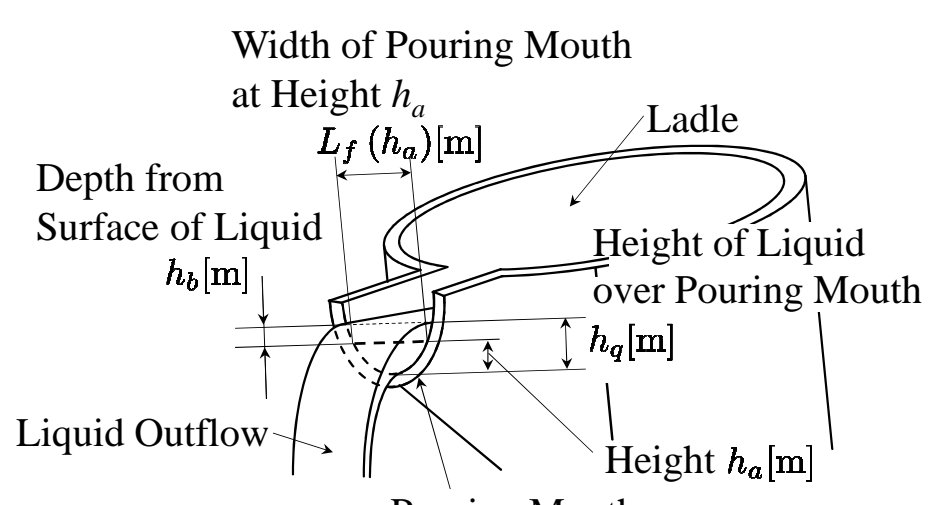

Pouring Mouth

Flow Rate Model: $P_{f}$. When the outflow process is shown in Fig. 4, the volume balance equation of pouring with a variation of $\omega$ becomes

$$
\dot{h}_{q}=-\frac{q(h)}{A(\theta)}-\frac{1}{A(\theta)}\left(\frac{\partial A(\theta)}{\partial \theta} h_{q}+\frac{\partial V_{s}(\theta)}{\partial \theta}\right) \omega .
$$


Now, if it is assumed that all of outflow comes from upper-side volume of a ladle, as shown in Fig. 5 , the relational equation of $h$ and $q$ is

$$
q\left(h_{q}\right)=c_{q} \int_{0}^{h_{q}} L_{f}\left(h_{b}\right) \sqrt{2 g h_{b}} d h_{b}\left(0<c_{q} \leq 1, h_{a}=h_{q}-h_{b}\right)
$$

based on Bernoulli's principle in the steady flow, where $L_{f}$ is a width at a height $h_{a}$ based on lower end of the pouring mouth, $h_{b}$ is a depth from a liquid surface at the pouring mouth, $c_{q}$ is a discharge coefficient identified by comparison of poured weights by experiment and simulation, and g is gravity acceleration $9.81 \mathrm{~m} / \mathrm{s}^{2}$.

\section{Mathematical Model of Sprue Cup: $P_{c}$}

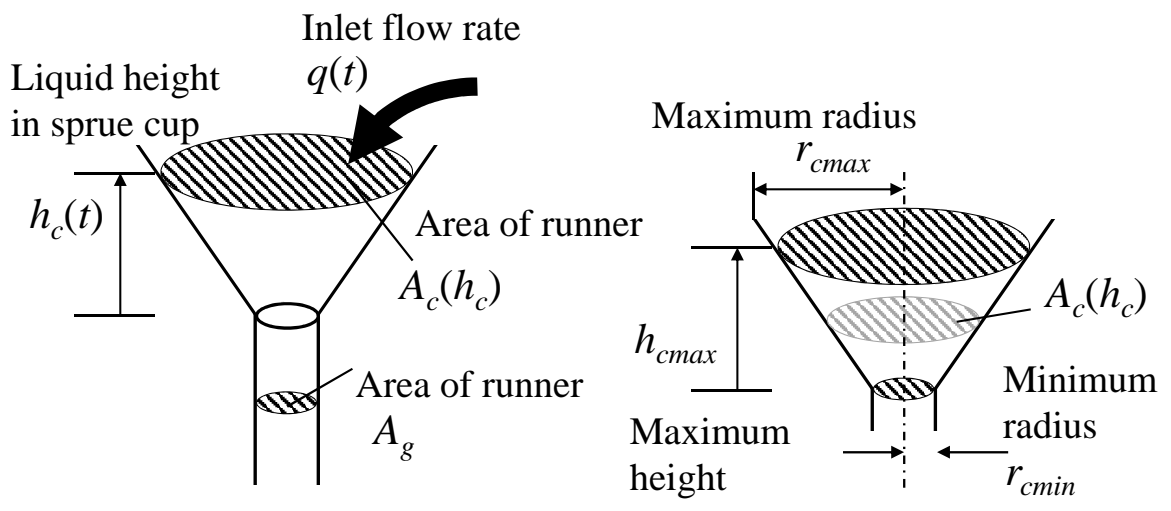

Fig. 6: Sprue cup shape in casting mold

In this study, the sprue cup shape of mold is defined as cone shape, as shown in Fig. 6. The mathematical model of sprue cup was derived as

$$
\dot{h}_{c}=\frac{q}{A_{c}\left(h_{c}\right)}+c_{c} \frac{A_{g}}{A_{c}\left(h_{c}\right)} \sqrt{2 g\left(h_{c}\right)}
$$

based on Bernoulli's principle. This is a dynamnical relation between liquid level in sprue cup $h_{c}$ and flow rate $q$. The cross-sectional area $A_{c}$ was descripted as the following function of $h_{c}$.

$$
A_{c}\left(h_{c}\right)=\pi r_{c}\left(h_{c}\right)^{2}=\pi\left\{\alpha_{1} h_{c}+\alpha_{0}\right\}^{2}, \alpha_{0}=r_{c m i n}, \alpha_{1}=\frac{r_{c m a x}-r_{c m i n}}{h_{c \max }}
$$

\section{Cascade Control System for Flow Rate and Mold Liquid Level}

Figures 7 and 8 shows the cascade control system proposed in this paper. This system is comprised by double feedback loop to enhance control response: inner loop was flowrate control system for pouring ladle, and outer loop was level control in sprue cup. Details of each loops are shown in the following.

Flow Rate Control. The process model of flow rate was described as nonlinear system. Therefore, the exact linearization method [12] was applied to construct good feedback system. Since the motor model $P_{t}$ has enough responsiveness, the dynamics of motor Eq. 1 was approximated by $\omega=K_{t} u_{t}[6]$ and the flow rate model was described as

$$
\dot{h}_{q}=-\frac{q(h)}{A(\theta)}-\frac{1}{A(\theta)}\left(\frac{\partial A(\theta)}{\partial \theta} h_{q}+\frac{\partial V_{s}(\theta)}{\partial \theta}\right) K_{t} u_{t}
$$




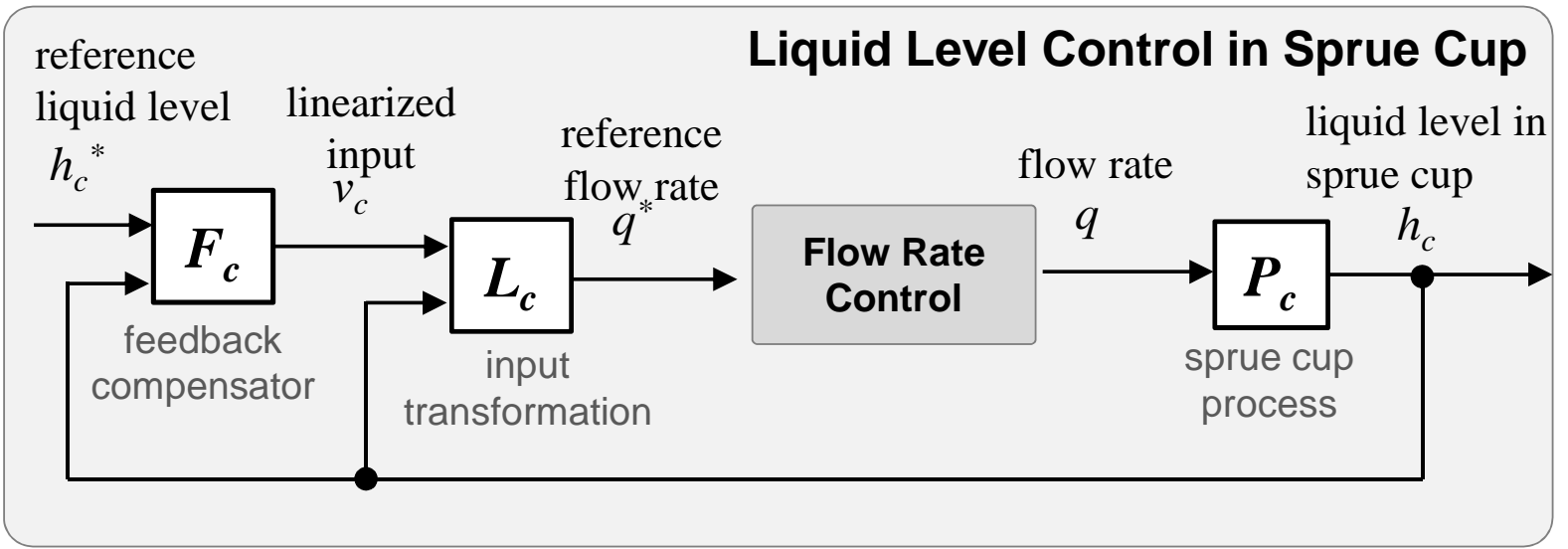

Fig. 7: Cascade control system for pouring flow rate and liquid level in sprue cup

reference reference motor

flow rate liquid height linearized voltage

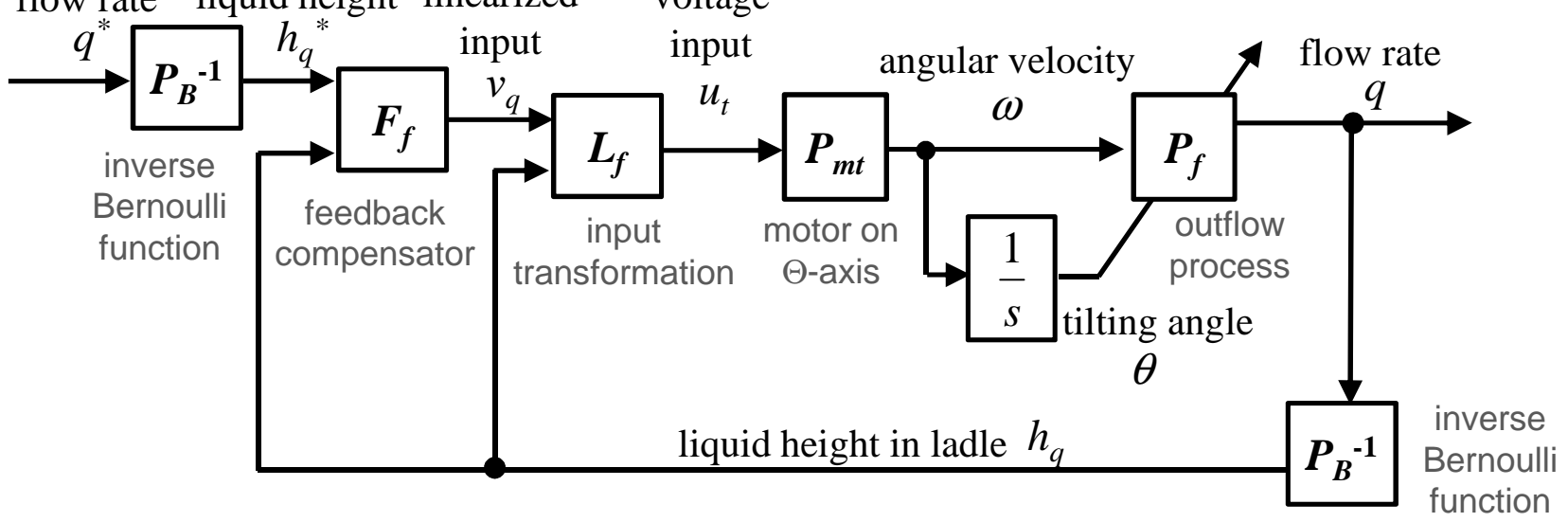

Fig. 8: Flow rate control loop of cascade control system

The input transformation of plant model was derived by rearrangement of Eq. 6 for $u_{t}$ and definition of the new input $\nu_{q}=\dot{h}_{q}$.

$$
u_{t}=-\frac{A(\theta) \nu_{q}+q(h)}{K_{t}\left(\frac{\partial V_{s}(\theta)}{\partial \theta}+\frac{\partial A(\theta)}{\partial \theta} h\right)}
$$

Finally, the feedback compensator was constructed to stabilize the linearized system.

$$
\nu_{q}=\dot{h}_{q}^{*}-k_{q}\left(h_{q}-h_{q}^{*}\right)
$$

where, $h_{q}^{*}$ is the reference liquid height in the pouring ladle and $k_{q}$ is the parameter of the feedback compensator. Exact linearization is not linear approximation, but system is exaxtly linearized by nonlinear transformation. Note that the reference liquid height $h^{*}$ was derived from inverse Bernoulli function of Eq. 3.

Liquid Level Control. The sprue cup model was also a nonlinear system. The exact linearization method was also applied as same as the flow rate control. The new input for the sprue cup model is defined as $\nu_{c}=\dot{h}_{c}$.

$$
q^{*}=A_{c}\left(h_{c}\right) \nu_{c}+c_{c} A_{g} \sqrt{2 g h_{c}}
$$


The feedback compensator was constructed in the following and its parameter $k_{c}$ is also defined.

$$
\nu_{c}=\dot{h}_{c}^{*}-k_{c}\left(h_{c}-h_{c}^{*}\right)
$$

\section{Simulation Results}
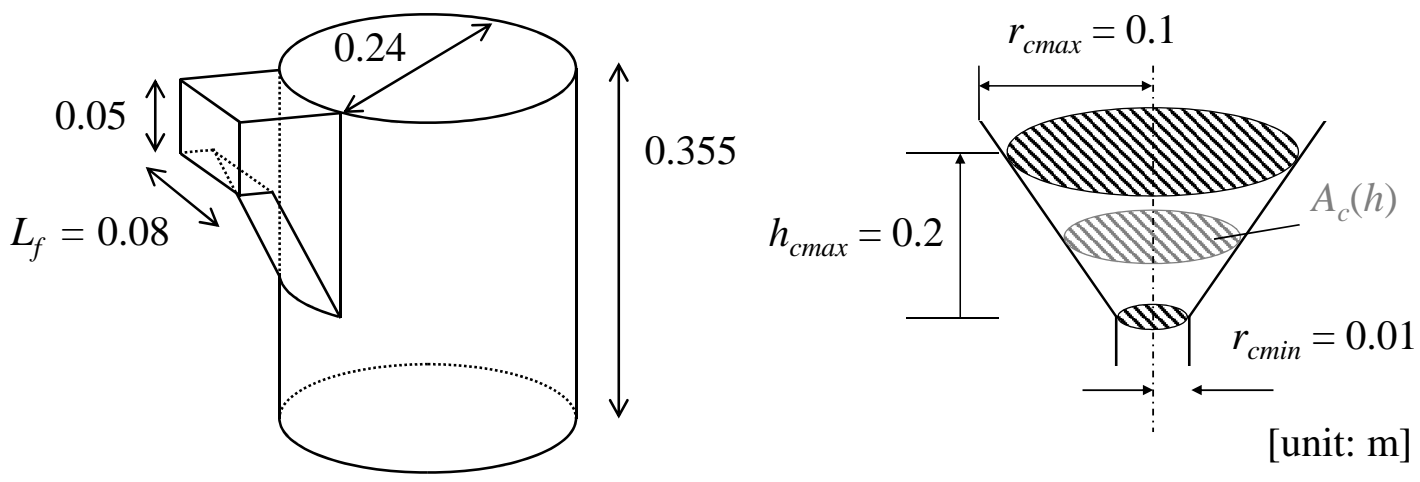

Fig. 9: Shapes of pouring ladle and mold sprue cup in simulations

The proposed system is verified by control simulations. The pouring ladle shape is assumed as Fig. 9. This cylindrical ladle has a rectangle mouth, therefore the Bernoulli function for $P_{f}$, Eq. 3 can be simplified as

$$
q=\frac{2}{3} c_{q} L_{f} \sqrt{2 g h_{q}^{3}}
$$

Also, parameters of ladle shapes related to Eq. 2 are given as Fig. 10.

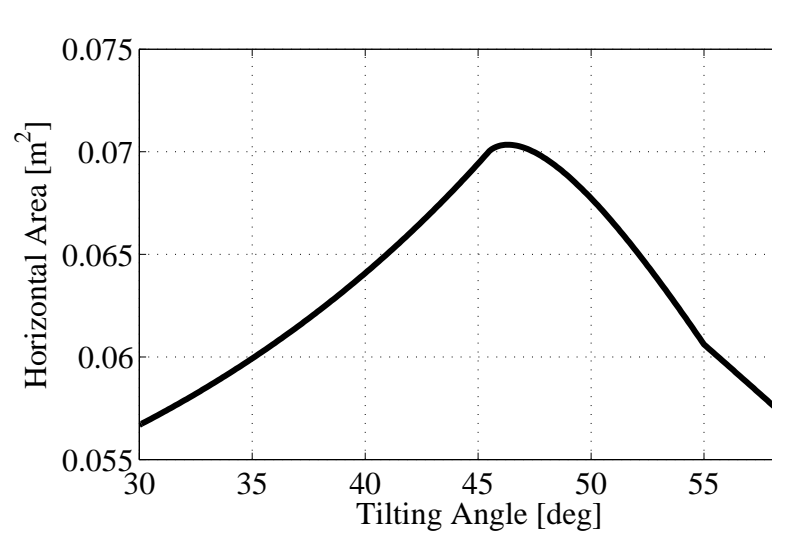

(a) $A(\theta)$

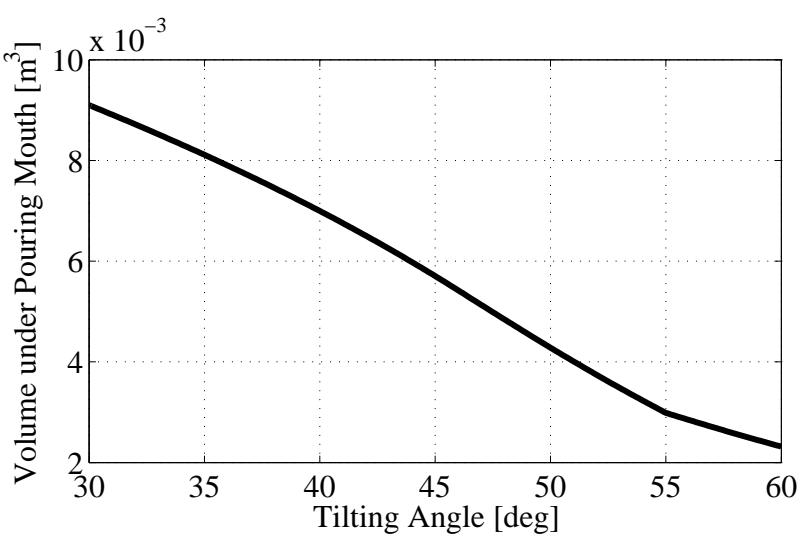

(b) $V_{s}(\theta)$

Fig. 10: Ladle shape parameters

Model parameters and controller parameters of each feedback loops are given as Table 1 in this paper.

Figure 11 shows control simulation results. This graphs show the comparison between references and control outputs. And, the case that each plant model has modeling error was also verified to check the robustness of the proposal control systems. In this paper, modeling errors were given with respect to the decreased discharge coefficients $c_{q}$ and $c_{c}$. Each decreased coefficient has $80 \%$ value of correct one. The slope of the reference liquid level $h_{c}^{*}$ was given by a fifth-order polynomial function. 
Table 1: Simulation parameters

\begin{tabular}{|l|c|}
\hline discharge coefficient in $P_{f}: c_{q}$ & 0.89 \\
\hline discharge coefficient in $P_{c}: c_{c}$ & 0.70 \\
\hline feedback gain of Eq. 8: $k_{q}$ & 100 \\
\hline feedback gain of Eq. 10: $k_{c}$ & 20 \\
\hline
\end{tabular}

In each result, the control results of flow rate and liquid level correspond reasonably well with the references. Also, the response of liquid level with modeling error is also closely matched to the response without modeling error. The flow rate and motor input with modeling error were reasonally reduced than model's correct case for model change.

Therefore, these simulation results show the effectiveness of the proposed cascade control system.

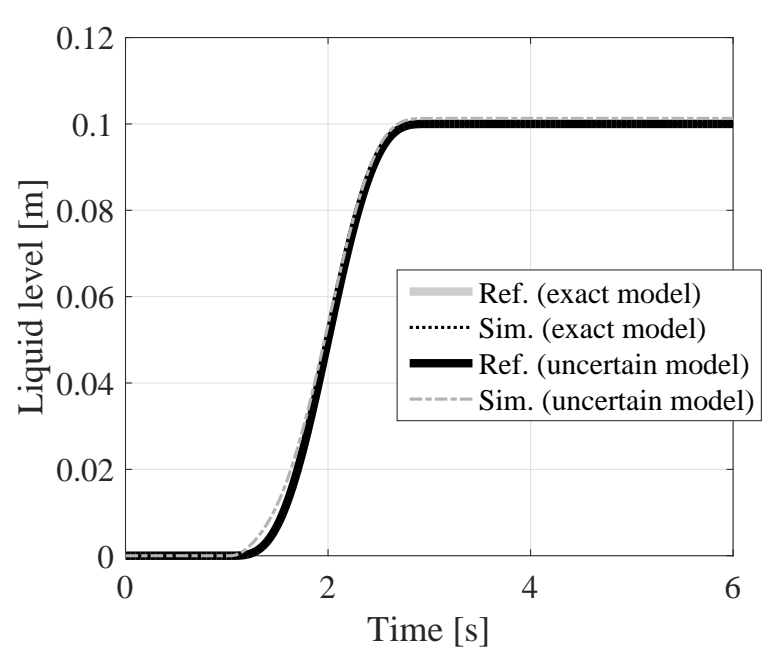

(a) Liquid level in sprue cup: $h_{c}$

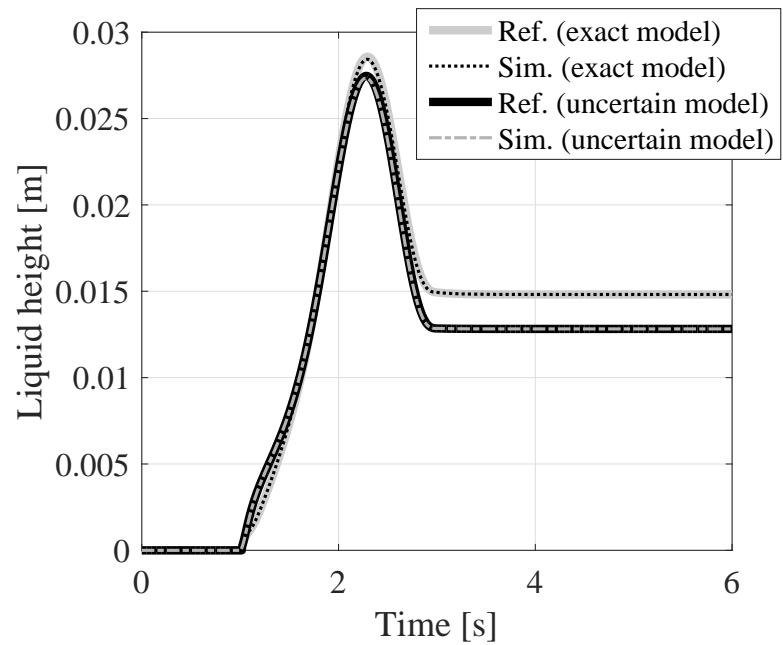

(c) Height of liquid over pouring ladle mouth: $h_{q}$

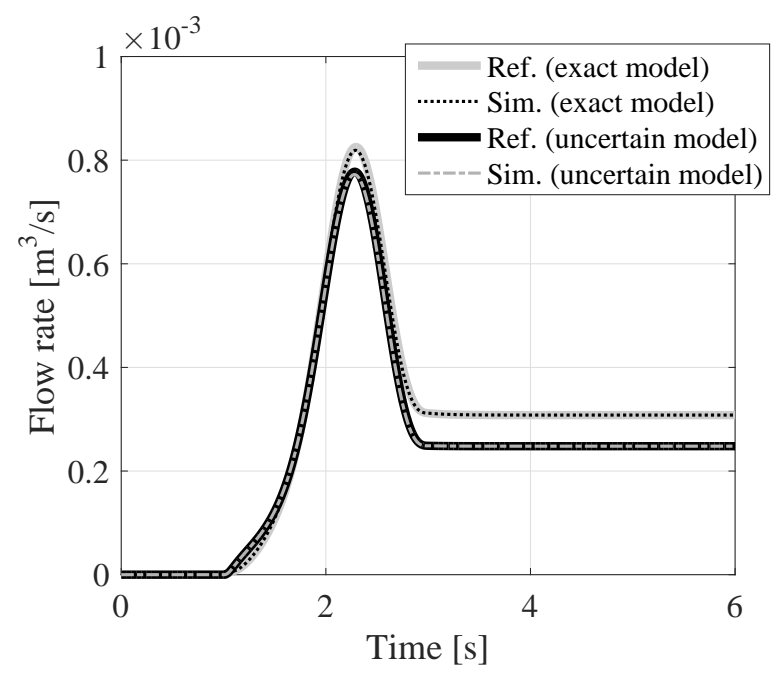

(b) Flow rate of pouring ladle: $q$

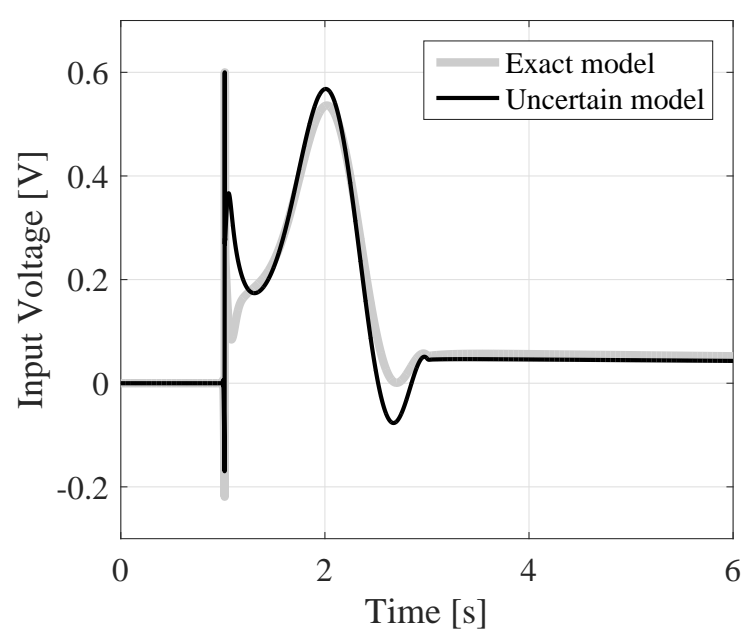

(d) Input voltage of tilting motor: $u_{t}$

Fig. 11: Simulation results of the proposed system 


\section{Summary}

In this paper, the cascade control system for level control of tilting-type automatic pouring system was proposed. This system had two feedback loops to control flow rate and liquid level in sprue cup. Each feedback system was constructed by model-based approach and used exact linearization method. The robust control system using the proposed control was validated by simulaiton results.

On going works, feedback system by visual measurement of liquid level control will be embedded to this control system and the effectiveness of proposed approach will be verified by experiments using the automatic pouring system in laboratory.

\section{References}

[1] K. Terashima: SOKEIZAI 39 (1998) p.1

[2] Y. S. Lerner: Modern Casting, No. 93, Vol. 11, (2003) p. 44

[3] K. Yano, M. Kaneko, Y. Sugimoto, and K. Terashima: Journal of the Robotics Society of Japan, Vol. 21 (2003) No. 6 p. 670

[4] K. Terashima, K. Yano, Y. Sugimoto, and M. Watanabe: 10th IFAC Symposium on Automation in Mining, Mineral and Metal Processing, (2001) p. 182

[5] Y. Noda and K. Terashima: Transactions of the Japan Society of Mechanical Engineers Series C Vol. 72 (2006) No. 722 p. 3147

[6] Y. Noda, M. Zeitz, O. Sawodny, and K. Terashima: Proceedings of 2011 IEEE International Conference on Control Applications, (2001), p. 1468

[7] A. Ito, Y. Noda, and K. Terashima: 2012 IEEE Multi-Conference on System and Control, (2012) p. 246

[8] M. Birkhold, C. Friedrich, and A. Lechler: IFAC-Papers On Line, Vol. 48, No. 17 (2015), p. 195

[9] E. Tabatabaei: Ductile Iron News, (2000), Issue No. 1

[10] S. Paranjape and P. D. Chaubal: Metalworld, June 2010, (2010) p. 24

[11] V. I. Dubodelov, V. K. Pogorsky, and M. S. Goryuk: Proc. 9th International Symposium on Science and Processing of Cast Iron, (2009) p. 481

[12] Jean-Jacques E. Slotine, Weiping Li: Applied Nonlinear Control (1990) 